\title{
LE PROBLÈME DE HELMHOLTZ POUR DES OBSTACLES PEU RÉGULIERS
}

\author{
Carole Erba, Régine Weber, et Michel Zinsmeister
}

\begin{abstract}
The classical Helmholtz model for wakes behind an obstacle in perfect fluid mechanics boils down in complex analysis into a Riemann problem with free boundary, the free boundary being the wake and being such that the Riemann map parametrizes it as arclength parametrization. We generalize a method due to Coifman and Meyer $[\mathbf{C M}]$ to solve this problem for a class of non-smooth obstacles.
\end{abstract}

\section{Introduction et énoncé du résultat}

Dans la théorie du fluide parfait incompressible plan, un écoulement permanent dans un domaine $\Omega$ est donné par une fonction holomorphe $f: \Omega \rightarrow \mathbb{C}$ dont la partie imaginaire est constante au bord.

Les parties réelles et imaginaires de $f$ sont usuellement notées $\Phi$ et $\Psi$ et sont appelées respectivement fonction potentiel et fonction courant.

Les lignes de niveau de $\Psi$ ne sont autres que les lignes de courant du fluide. Les trajectoires orthognonales à ces lignes de courant sont les équipotentielles $\{\Phi=$ cste $\}$.

La dérivée $\omega(z)=f^{\prime}(z)$ est appelée vitesse complexe. Si l'on munit le plan complexe de sa structure euclidienne canonique, la vitesse de l'écoulement, qui n'est autre que $\vec{V}=\nabla \Phi$, est encore égale à $\bar{\omega}$.

Si maintenant $\Omega^{\prime}$ est un autre domaine de $\mathbb{C}$ conformément équivalent à $\Omega$, alors la donnée d'une représentation conforme $h: \Omega^{\prime} \rightarrow \Omega$ permet de définir un écoulement $g$ dans $\Omega^{\prime}$ par $g=f \circ h$.

Ainsi l'écoulement uniforme $f(z)=z$ dans le demi-plan supérieur $\mathbb{R}_{+}^{2}=$ $\{x+i y ; y>0\}$ permet de définir un écoulement naturel dans tout domaine à gauche d'une courbe de Jordan $\Gamma=\{z=\gamma(t), t \in \mathbb{R}\}$ avec $|\gamma(t)| \rightarrow \infty$ quand $|t| \rightarrow+\infty$, par $g(\xi)=f(h(\xi))$ où $h$ est une représentation conforme du domaine à gauche de $\Gamma$ sur $\mathbb{R}_{+}^{2}$ telle que $h(\infty)=\infty$.

2000 Mathematics Subject Classification. 32A37, 30C20, 76B10.

Key words. Helmholtz Wake, BMOA, Riemann mapping theorem, free boundary. 
Vue sous cet angle, la théorie des fluides parfaits plan apparaît comme équivalente à la théorie de la représentation conforme de Riemann.

Un autre exemple illustrant cette hypothèse est celui d'un écoulement autour d'un obstacle.

Cet obstacle est un compact $K$ connexe plein du plan et $\mathbb{C} \backslash K$ peut être transformé conformément en $\mathbb{C} \backslash[-1,1]$ par une transformation préservant $\infty$.

On peut alors transférer l'écoulement uniforme $f(z)=z$ sur $\mathbb{C} \backslash[-1,1]$ en un écoulement sur $\mathbb{C} \backslash K$ via cette représentation confome.

On pourrait ainsi fabriquer un écoulement autour d'un segment vertical orthogonal à la vitesse à l'infini. Mais il est clair qu'un tel écoulement ne correspondrait pas à la réalité. Quiconque s'est attardé à observer le passage de l'eau autour d'un pilier de pont a pu observer l'existence d'un sillage entourant une zone de fluide mort.

Helmholtz a construit un tel modèle de sillage : il est délimité par deux courbes joignant les points de décollement qui doivent être connus, et le domaine constitué du complémentaire de la zone bordée par le sillage est l'image d'une représentation conforme $h$ définie sur $\mathbb{C} \backslash \mathbb{R}_{+}$qui est telle que sur les deux segments correspondant aux deux branches du sillage (il faut voir $\mathbb{R}_{+}$comme dédoublé, c'est à dire avec un bord supérieur et un bord inférieur) la représentation $h$ vérifie $\left|h^{\prime}\right|=V_{0}$, la vitesse scalaire à l'infini. On peut toujours normaliser le problème de sorte que $V_{0}=1$. L'hypothèse peut alors se paraphraser en disant que $h$ réalise une paramétrisation par la longueur d'arc des deux branches du sillage.

Naturellement il n'est pas clair que ce problème ait une solution. Nous l'appellerons problème de Helmholtz et ce problème peut être vu comme un problème de Riemann avec frontière libre.

Ce problème a été étudié mathématiquement en 1935 par J. Leray [L] qui a obtenu des résultats d'existence pour des obstacles de classe $C^{1+\alpha}$ avec $\alpha>\frac{1}{2}$.

Nous proposons ici une approche différente dans un cadre simplifié en utilisant l'approche de Coifman et Meyer $[\mathbf{C M}]$ du théorème de Riemann.

Cette approche nous permettra de réduire les hypothèses de régularité sur l'obstacle.

Afin de comprendre la simplification que nous opérons, considérons le rôle de l'origine 0 dans le problème de Helmholtz.

Dans la représentation conforme, ce point correspond au point de séparation de l'obstacle, les lignes de courant aboutissant au dessus de ce point glissent vers le haut le long de l'obstacle tandis que les autres 
glissent vers le bas. Ce point ne peut être fixé arbitrairement et il s'agit d'une des difficultés du problème. Il est néanmoins un cas où ce point est clairement défini : c'est le cas d'un obstacle symétrique par rapport à $\mathbb{R}$. Le point de séparation est alors l'intersection de l'obstacle avec l'axe réel et la ligne de courant séparante est la demi droite négative issue de ce point.

En remplaçant l'obstacle par la réunion de l'obstacle et de cette ligne de courant séparante, on est alors ramené à un problème simplement connexe. C'est dans ce contexte que nous nous placerons. On rappelle qu'une courbe de Lavrentiev passant par l'infini est une courbe de Jordan localement rectifiable dont la représentation par la longueur d'arc $z(s)$, $s \in \mathbb{R}$ vérifie

(1) $\lim _{s \rightarrow \infty}|z(s)|=\infty$.

(2) $\exists C>0 / \forall s_{1}, s_{2} \in \mathbb{R}\left|s_{1}-s_{2}\right| \leq C\left|z\left(s_{1}\right)-z\left(s_{2}\right)\right|$.

Nous appellerons norme de la courbe la plus petite constante $C>0$ telle que (2) soit vérifiée. Avant de poursuivre il nous faut discuter de quelques préliminaires.

Soit $I$ un intervalle fermé de $\mathbb{R}$. L'espace $B M O(I)$ est l'espace des fonctions $b \in L_{\text {loc }}^{1}(I)$ telles que

$$
\|b\|_{*}=\sup _{J \subset I} \frac{1}{|J|} \int_{J}\left|b(x)-\frac{1}{|J|} \int_{J} b(t) d t\right| d x<\infty .
$$

Muni de $\|\cdot\|_{*}$, cet espace est un espace de Banach modulo les constantes.

Il est clair que si $b \in B M O(\mathbb{R})$ alors $b_{\mid I} \in B M O(I)$.

Réciproquement, si $b \in B M O(I)$, comment peut-on la prolonger en une fonction de $B M O(\mathbb{R})$ ?

Intéressons nous plus spécialement au cas de $I=\mathbb{R}_{+}$.

Soit $a$ une fonction définie sur $\mathbb{R}_{+}$. Considérons trois extensions possibles à $\mathbb{R}$ :

$$
\begin{gathered}
\tilde{a}(x)= \begin{cases}a(x), & \text { si } x \geq 0 \\
0, & \text { si } x<0\end{cases} \\
a_{\text {pair }}(x)= \begin{cases}a(x), & \text { si } x \geq 0 \\
a(-x), & \text { si } x<0\end{cases} \\
a_{\text {impair }}(x)= \begin{cases}a(x), & \text { si } x \geq 0 \\
-a(-x), & \text { si } x<0 .\end{cases}
\end{gathered}
$$


Notons que $a_{\text {pair }}+a_{\text {impair }}=2 \tilde{a}$. Par ailleurs il est facile de se rendre compte que $a_{\text {pair }} \in B M O(\mathbb{R})$ dès que $a \in B M O\left(\mathbb{R}_{+}\right)$.

On en déduit immédiatement que $\tilde{a} \in B M O(\mathbb{R}) \Longleftrightarrow a_{\text {impair }} \in B M O(\mathbb{R})$. Mais cette condition n'est pas obligatoirement satisfaite, comme le montre l'exemple $a(x)=\ln (x), x \geq 0$.

Nous désignons par $E$ l'espace des fonctions $a \in B M O\left(\mathbb{R}_{+}\right)$telles que $\tilde{a} \in B M O(\mathbb{R})$, muni de la norme $\|\tilde{a}\|_{*}$. En d'autres termes, $E$ est isométrique à l'espace des fonctions $b \in B M O(\mathbb{R})$ qui s'annulent sur $\mathbb{R}^{-}$, qui est clairement un fermé de $B M O(\mathbb{R})$. On peut aussi voir $E$ comme le sous-espace de $B M O(\mathbb{R})$ des fonctions impaires.

Revenons au courbes de Lavrentiev. Si $z(s)$ est la paramétrisation par longueur d'arc de l'une d'entre elles, alors on peut montrer ([CM]) qu'il existe $\alpha \in B M O(\mathbb{R})$ telle que

$$
z^{\prime}(s)=e^{i \alpha(s)}, \quad s \in \mathbb{R}
$$

et que réciproquement si $\|\alpha\|_{*}$ est assez petit alors $z(t)=\int_{0}^{t} e^{i \alpha(s)} d s$ paramétrise une courbe de Lavrentiev de norme proche de 1.

Nous sommes maintenant en mesure d'énoncer le résultat principal de ce travail :

Théorème 1.1. Il existe $\epsilon_{0}>0$ tel que si $\alpha \in B M O\left(\mathbb{R}_{+}\right)$et $\|\alpha\|_{*}<\epsilon_{0}$ alors $\Gamma_{\alpha}$ se prolonge en une courbe $\Gamma$ de Lavrentiev de norme proche de 1 et qui est telle qu'il existe $F: \mathbb{R}_{+}^{2} \rightarrow \Omega$ (le domaine à gauche de $\Gamma$ ), une représentation conforme telle que $F(0)=0, F(\infty)=\infty$ et $F_{\mid]-\infty, 0[}$ est une paramétrisation de $\Gamma \backslash \Gamma_{\alpha}$ par longueur d'arc.

\section{Preuve du théorème}

Supposons le problème résolu. Alors (voir $[\mathbf{Z}]$ ) $\log \left|F^{\prime}\right| \in B M O(\mathbb{R})$ et donc, puisque $\left|F^{\prime}\right|=1$ sur $\mathbb{R}_{-},\left.\log \left(F^{\prime}\right)\right|_{\mathbb{R}_{+}} \in E$. Par ailleurs $z$ et $F$ représentent deux paramétrisations de $\Gamma_{\alpha}$ par $\mathbb{R}_{+}$: on passe donc de l'une à l'autre par composition par un homéomorphisme de $\mathbb{R}_{+}$, soit $F(x)=z(h(x))$. On a donc $h^{\prime}=|F|$ et $\log h^{\prime} \in E$. On a aussi

$$
\operatorname{Arg} F^{\prime}=\alpha \circ h \quad \operatorname{sur} \mathbb{R}_{+} \text {. }
$$

Appelons $H$ la transformée de Hilbert sur $\mathbb{R}$ normalisée de sorte que pour une fonction holomorphe dans $\mathbb{R}_{+}^{2}$, la partie imaginaire au bord soit la transformée de Hilbert de la partie réelle. En particulier

$$
\operatorname{Arg} F^{\prime}=H\left(\log \left|F^{\prime}\right|\right)
$$

et (1) devient

$$
\left.H\left(\log h^{\prime}\right)\right|_{\mathbb{R}_{+}}=\alpha \circ h .
$$


Posons $\theta=h^{-1}$ et désignons par $V_{\theta}$ l'opérateur $: b \mapsto b \circ \theta$ qui est un isomorphisme de $B M O(\mathbb{R})$ et donc aussi de $E$ puisque $V_{\theta}$ et $V_{h}$ fixent $E$. Comme $h^{\prime}=\frac{1}{\theta^{\prime} \circ \theta^{-1}}$, l'équation (2) devient

$$
\alpha=-\left.V_{\theta} H V_{\theta}^{-1}\left(\log \theta^{\prime}\right)\right|_{\mathbb{R}_{+}} .
$$

Pour faire la synthèse, on raisonne comme dans $[\mathbf{C M}]$. $\alpha$ étant donné, si l'on parvient à résoudre (3) c'est à dire à trouver $\log \theta^{\prime} \in E$ pour lequel (3) est vérifié alors

$$
F(x)=\int_{0}^{x} \exp \left\{\log h^{\prime}(u)+i H\left(\log h^{\prime}\right)\right\} d u
$$

est la fonction cherchée (ou plutôt sa trace sur $\mathbb{R}$ ). Pour résoudre (3), on reprend l'idée de $[\mathbf{C M}]$ (qui est déjà celle de $[\mathbf{L}]$ ) : on se ramène à un problème de point fixe. Pour le faire, nous aurons besoin du lemme suivant :

Lemme 2.1. L'opérateur $T: b \mapsto 1_{[0, \infty[} H(b)$ est un isomorphisme de $E$ sur $B M O\left(\mathbb{R}_{+}\right)$.

Preuve du lemme: Montrons que $T$ est injectif : si $b \in E$ est tel que $T(b)=0$, alors $b H(b)$ est identiquement nulle et donc $(b+i H(b))^{2}$ est une fonction réelle, donc constante puisque valeur au bord d'une fonction holomorphe. Donc $b+i H(b)$ est aussi constante et donc nulle puisque $b=0$ sur $\mathbb{R}_{-}$et $H b=0$ sur $\mathbb{R}_{+}$.

Pour montrer que $T$ est surjectif, donnons nous $\beta \in B M O\left(\mathbb{R}_{+}\right)$. On cherche $a \in E$ tel que $T(a)=\beta$. Considérons la transformée de Cauchy de $a$. C'est une fonction holomorphe sur $\mathbb{C} \backslash \mathbb{R}_{+}$et les formules de Plemmelj $([\mathbf{Z}])$ montrent que cette fonction a pour valeur au bord positive sur $\mathbb{R}_{+}, \frac{1}{2}(a+i H a)$ et pour valeur au bord négative, $\frac{1}{2}(-a+i H a)$.

Mais si $\zeta \mapsto F(\zeta)$ est une fonction holomorphe sur $\mathbb{C} \backslash \mathbb{R}_{+}$, alors $z \mapsto$ $F\left(z^{2}\right)$ est holomorphe sur $\mathbb{R}_{+}^{2}$. On en déduit qu'il existe une fonction holomorphe sur $\mathbb{R}_{+}^{2}$ dont les valeurs au bord sont

$$
\begin{array}{ll}
\frac{1}{2}\left(a\left(x^{2}\right)+i H a\left(x^{2}\right)\right), & x \geq 0 \\
\frac{1}{2}\left(-a\left(x^{2}\right)+i H a\left(x^{2}\right)\right), & x<0 .
\end{array}
$$


Posons alors

$$
A(x)= \begin{cases}a\left(x^{2}\right), & \text { si } x>0 \\ -a\left(x^{2}\right), & \text { si } x \leq 0\end{cases}
$$

et

$$
B(x)= \begin{cases}H a\left(x^{2}\right), & \text { si } x \geq 0 \\ H a\left(x^{2}\right), & \text { si } x<0\end{cases}
$$

et l'on a $A=-H(B), B=H(A)$.

Pour effectuer la synthèse posons maintenant

$$
B(x)= \begin{cases}\beta\left(x^{2}\right), & \text { si } x \geq 0 \\ \beta\left(x^{2}\right), & \text { si } x<0\end{cases}
$$

et

$$
A(x)=-H(B), \quad a(x)=A(\sqrt{x}), \quad x>0 .
$$

Le fait que $A$ soit impaire et dans $B M O$ prouve que $a \in E$ car $f \mapsto$ $(x \mapsto f(\sqrt{x}))$ préserve $B M O(\mathbb{R})([\mathbf{Z}])$. Enfin le fait que $B=H(A)$ prouve que $\beta=T(a)$.

Revenons à la preuve du théorème. On peut réécrire (3) sous la forme

$$
\alpha=-V_{\theta} T V_{\theta}^{-1}\left(\log \theta^{\prime}\right) .
$$

Posons $F(b)=-V_{\theta} T V_{\theta}^{-1}(b)$ où $\log \theta^{\prime}=b$.

Si $\|b\|_{*}$ est assez petit, $V_{\theta}$ est alors un isomorphisme de $B M O([\mathbf{Z}])$. Posons encore

$$
\epsilon(b)=F(b)+T(b) .
$$

Alors (4) équivaut à $-T(b)+\epsilon(b)=\alpha$ ou encore

$$
-T^{-1}(\alpha)+T^{-1} \quad \text { o } \quad \epsilon(b)=b .
$$

Posons alors $\Lambda(b)=-T^{-1}(\alpha)+T^{-1}(\epsilon(b))$. On a

$$
\|\Lambda(b)-\Lambda(\beta)\|_{*} \leq C\|\epsilon(b)-\epsilon(\beta)\|_{*},
$$

et

$\|\epsilon(b)-\epsilon(\beta)\|_{*}=\left\|\left(T-V_{\theta} T V_{\theta}^{-1}\right)(b-\beta)-V_{\theta} T V_{\theta}^{-1}(\beta)+V_{\zeta} T V_{\zeta}^{-1}(\beta)\right\|_{*}$, où $\log \zeta^{\prime}=\beta$

$$
\leq C\left(\|b\|_{*}+\|\beta\|_{*}\right)\|b-\beta\|_{*}
$$

par les résultats de $[\mathbf{C M}]$ qui s'appliquent par restriction. 
Fixons alors $r>0$ tel que si $\|b\|_{*},\|\beta\|_{*}<r$ alors

$$
\|\Lambda(b)-\Lambda(\beta)\|_{*} \leq \frac{1}{2}\|b-\beta\|_{*} .
$$

Pour conclure, il suffit de montrer qu'il existe $\epsilon_{0}>0$ tel que si $\|\alpha\|_{*} \leq$ $\epsilon_{0}$ alors la boule fermée de $E$ de centre 0 et de rayon $r$ est invariante par $\Lambda$.

Mais $\|\epsilon(b)\|_{*} \leq C\|b\|_{*}^{2}$ par $[\mathbf{C M}]$, et il suffit de choisir $\epsilon_{0}$ tel que

$$
C \epsilon_{0}+C^{\prime} r^{2} \leq r
$$

ce qui est possible si $C^{\prime} r<1$ et $\epsilon_{0} \leq \frac{r\left(1-C^{\prime} r\right)}{C}$.

Ce qui précède s'applique en particulier au cas d'un obstacle de type diédral symétrique par rapport à l'axe réel dont l'intersection avec le demi plan supérieur est donnée par $\{y=f(x)\}$ où $f$ est une fonction lipschitzienne de petite norme sur $[0,1]$ nulle en 0 et $>0$ sur $] 0,1]$. Il suffit pour s'en convaincre d'appliquer ce qui vient d'être vu à la demi-courbe de Jordan définie par le demi axe réel négatif prolongé par le graphe de la fonction $f$. Le cas du dièdre correspond à $f(x)=x \tan \theta$. Dans ce cas, la solution est explicite et donnée par $F(z)=\lambda \Psi(z / \lambda)$ où $\lambda$ est tel que $|\lambda \Psi(1)|$ est égal à la longueur du côté du dièdre et

$$
\Psi^{\prime}(z)=\left(\frac{\sqrt{z}}{\sqrt{z-1}-i}\right)^{\frac{2 \theta}{\pi}} .
$$

\section{Références}

[CM] R. Coifman et Y. Meyer, Le théorème de Calderón par les "méthodes de variable réelle", C. R. Acad. Sci. Paris Sér. A-B 289(7) (1979), A425-A428.

[L] J. LERAY, Les problèmes de représentation conforme d'Helmholtz; théories des sillages et des proues, Comment. Math. Helv. 8(1) (1935-36), 250-263.

[Z] M. Zinsmeister, "Domaines de Lavrent'iev", Publications Mathématiques d'Orsay 85-3, Université de Paris-Sud, Département de Mathématiques, Orsay, 1985. 
Carole Erba:

MAPMO, UMR 6628

Université d'Orléans

BP 6759

Orléans cedex 2

France

E-mail address: carole.erba@univ-orleans.fr

Régine Weber:

Institut PRISME

Université d'Orléans

BP 6749

45067 Orléans cedex 2

France

E-mail address: regine.weber@univ-orleans.fr

Michel Zinsmeister:

MAPMO, UMR 6628

Université d'Orléans

BP 6759

Orléans cedex 2

France

E-mail address: zins@univ-orleans.fr

Primera versió rebuda el 23 de desembre de 2008, darrera versió rebuda el 29 de juliol de 2010. 